\title{
Dal controllo alla creazione del valore
}

\author{
Giulio Greco, Giuseppe D'Onza*
}

\begin{abstract}
This article reviews the research papers published on this issue, framing the topics within the research streams of the Management Control journal. In particular, this editorial starts with an overview of the recent evolution of the firm's control system. The evolution is oriented toward an increasing integration, covering governance, audit, risk and performance. Also, the evolution is linked to an increasing external projection of the firm's control system. The articles presented in the issue put into evidence this pattern and highlight the ways in which the firm's control system contributes to the value creation process.

The first paper provides an interesting theoretical discussion of the firm as intelligent living being. By conceptually developing the analogy, the paper discusses how the concept of homeostasis can help interpret the firm's control system, as aimed at preserving and fostering value creation. A group of three papers deals with the communication of the value created through external reporting. These papers highlight how the external reporting is used by a range of stakeholders to control the value created by the firm, where the concept of value develops from the financial perspective to the sustainability perspective. Two other papers study how the firm's control system produces the information on costs and performance, used for internal decisional needs. The issue is closed by two papers investigating the risk management system. These papers highlight how digital technologies and big data can help treating information useful to control the firm's relationships with external subjects.
\end{abstract}

Keywords: value, value creation, integrated control, GARP.

* Università di Pisa, Dipartimento di Economia e Management, giulio.greco@unipi.it, giuseppe.donza@unipi.it.

Management Control (ISSN 2239-0391, ISSNe 2239-4397), 2020, 2

DOI: 10.3280/MACO2020-002001

Copyright $\odot$ FrancoAngeli

N.B: Copia ad uso personale. È vietata la riproduzione (totale o parziale) dell'opera con qualsiasi mezzo effettuata e la sua messa a disposizione di terzi, sia in forma gratuita sia a pagamento. 
In questo numero della rivista Management Control continua il percorso di approfondimento sull'evoluzione dell'economia aziendale contemporanea e sui temi di dibattito attuale tra gli studiosi. In particolare, in questo issue ci soffermiamo sul sistema di controllo e approfondiamo le modalità in cui contribuisce al processo di creazione di valore.

Il sistema di controllo ha seguito alcune importanti linee di evoluzione negli ultimi anni. Una linea di evoluzione è costituita dall'integrazione delle componenti della Governance, dell'Audit, del Risk e della Performance, attraverso lo sviluppo di un modello integrato che denomineremo GARP. Questo modello integra le attività di controllo svolte nell'area della Governance, con quelle che fanno riferimento all' Audit sia interno che esterno, alla gestione dei rischi (Risk management) sviluppata secondo un approccio sistemico e alla Valutazione delle performance, attraverso dei modelli multidimensionali (D’Onza, 2008; Greco et al., 2019). Tra gli elementi qualificanti del modello GARP si evidenzia l'integrazione tra risk management e performance management, ai livelli strategico, direzionale e operativo.

L'integrazione che, oggi non è presente in moltissime aziende, può riguardare aspetti differenti dei sistemi di management quali la pianificazione strategica, per favorire lo sviluppo di piani industriali in condizioni di elevata incertezza e supportare le decisioni assunte con analisi di sensitività, di scenario o di stress testing; il reporting direzionale, per poter ponderare le performance realizzate con l'evoluzione dell'esposizione al rischio dell'azienda nel suo complesso e nei vari segmenti in cui si articola la sua attività oppure lo sviluppo di una cultura aziendale, fondata su una gestione diffusa del rischio nell'organizzazione, per supportare l'adozione di politiche gestionali che bilancino il miglioramento delle performance con i rischi che l'azienda si assume con intraprendere nuove iniziative a livello operativo. In questo modo, l'integrazione può favorire lo sviluppo di uno strumento di controllo della creazione del valore da parte dell'alta direzione, degli organi di governo societario e di altri attori interni all'azienda e favorire lo sviluppo di sistemi di reporting per l'esterno volte a rafforzare l'accountability rispetto ai diversi portatori di interessi.

Un'altra linea di evoluzione dei sistemi di controllo è legata alla crescente proiezione esterna, a tutela degli interessi della vasta platea di stakeholder aziendali. Anche nelle aziende for profit, si sviluppa infatti l'esigenza che i sistemi di controllo integrino la dimensione economica e quella sociale, andando a soddisfare esigenze di conoscenza esterne e di sviluppo di relazioni cooperative con i fornitori, $\mathrm{i}$ clienti, i finanziatori, i dipendenti e, in generale 
con la comunità in senso ampio (Mancini, 1999; Bagnoli, 2010; Paolini, 2018).

Il presente numero della rivista include articoli che si inseriscono, per le tematiche trattate, nel percorso sopra delineato.

L'articolo di Catturi e Ricci Paulesu mette in evidenza l'azione del sistema di controllo interno nella creazione e nella conservazione del valore in termini di equilibrato sviluppo aziendale. In particolare, il lavoro tratta dell'azienda come "organismo intelligente". L'articolo utilizza la metafora azienda-organismo vivente nell'interpretazione dei rapporti tra l'ambiente interno e quello esterno. Gli Autori analizzano il sistema di controllo come strumento di raggiungimento e conservazione di una situazione di sviluppo armonico e coordinato tra ambiente interno ed esterno, affine all'omeostasi cellulare, che viene ripristinata ogni qualvolta le turbolenze esterne causino un disequilibrio.

Le riflessioni proposte nell'articolo sono particolarmente interessanti e di attualità, poiché nello scenario odierno in cui l'emergenza sanitaria ha destabilizzato il funzionamento dell'ambiente esterno, diviene fondamentale assumere decisioni che devono rispristinare l'equilibrio funzionale degli organi aziendali ed evitare che le turbolenze esterne possano comprometterlo. In questa prospettiva, i sistemi integrati GARP possono svolgere un ruolo importante per supportare le scelte volte a ripensare il business model delle aziende ed avviarle verso nuove traiettorie di sviluppo.

I tre lavori successivi trattano del tema della creazione di valore con riferimento alla comunicazione con l'esterno. Essi mettono in evidenza il controllo esercitato sul processo di creazione di valore dai soggetti esterni all'azienda, attraverso la rendicontazione per l'esterno. Tra i soggetti non vi sono solo gli investitori, interessati prevalentemente alla comunicazione economico-finanziaria, ma anche più in generale gli stakeholder interessati alla creazione di valore in termini di sostenibilità sociale e ambientale.

Il lavoro di Garzella, Capurro, Fiorentino e Morrone esplora il ruolo della comunicazione economico-finanziaria nell'ambito del processo di creazione di valore. Gli Autori ritengono che l'ampliamento della comunicazione alle informazioni sul valore del capitale economico rivesta un ruolo importante per il controllo dell'operato del management e per la rendicontazione dei risultati ottenuti.

L'articolo di Madonna, Cestari e Callegari analizza la value relevance dell'informazione di bilancio per i mercati finanziari. Gli Autori mostrano empiricamente come le informazioni che gli investitori ritengono maggiormente rilevanti siano quelle legate agli intangibili e ai dati su future prospet- 
tive di crescita. Appare quindi come gli investitori siano alla ricerca di informazioni sui driver della creazione di valore, andando oltre i dati classici legati all'utile di esercizio.

Nel suo articolo, Hristov tenta di incardinare concettualmente il tema della sostenibilità nella creazione di valore. L'Autore pone in evidenza come il sistema di controllo sia fondamentale per ampliare il concetto di valore creato alle dimensioni delle sostenibilità sociale, ambientale e culturale. La promozione di una cultura sostenibile all'interno e all'esterno dell'azienda viene considerata come un obiettivo per il sistema di controllo aziendale e come elemento di creazione di valore sostenibile nel lungo termine.

Gli articoli che seguono - di Del Bene e Sanna, e di Leotta, Rizza e Ruggeri- , mettono in evidenza l'azione del sistema di controllo nella creazione di valore per l'interno dell'azienda. Essi studiano come il sistema di controllo metta a disposizione le informazioni, in particolare, sui costi e sulla performance, utili per le decisioni interne.

In particolare, l'articolo di Del Bene e Sanna pone in evidenza le criticità nell'applicazione della contabilità analitica nelle aziende sanitarie. Al tempo stesso, esso mette in luce le potenzialità dell'uso dello strumento in un'ottica di miglioramento della gestione, delle relazioni organizzative e stimolo all'assunzione di comportamenti ottimali da parte dei responsabili, nelle prospettive clinico-assistenziale, organizzativa e di utilizzo delle risorse.

Il lavoro di Leotta, Rizza e Ruggeri si sofferma sul reporting nell'ambito del sistema di controllo di gestione delle PMI. Utilizzando un approccio di ricerca interventista, la ricerca empirica mostra il contributo dei soggetti interni all'azienda nella costruzione del sistema di reporting. Il contributo dei vari attori appare determinante nella strutturazione di un sistema efficace in grado di soddisfare i diversi fabbisogno informativi.

Chiudono l'issue due lavori sul tema del risk management, visto come elemento del controllo delle relazioni tra azienda e soggetti esterni all'azienda. In particolare, i lavori mettono in luce il trattamento sistematico delle informazioni provenienti dall'esterno per il controllo relazionale, rispetto al quale le nuove tecnologie e i big data possono offrire prospettive importanti di sviluppo.

Il lavoro di Mancini e Presti esamina l'utilizzo dei social media nell'attività di risk management. L'articolo fornisce una review della letteratura e contiene proposte di ricerche future lungo due direttrici: la relazione tra risk management e social media management; le fasi del processo di risk management nelle quali il social media management può essere efficacemente utilizzato. 
L'articolo di Moro Visconti esamina l'utilizzo delle tecnologie digitali per la gestione dei rapporti con gli stakeholder aziendali, nell'ambito del sistema di governance. L'Autore applica la network theory e intepreta gli stakeholder aziendali come dei nodi, con i quali condividere informazioni e cosviluppare valore nell'ambito di un eco-sistema digitale sostenibile. In questa prospettiva, l'utilizzo dei big data consente ridurrebbe le asimmetrie informative e i rischi relazionali.

\section{References}

Chenhall R.H. (2003), Management control systems design within its organizational context: findings from contingency-based research and directions for the future, Accounting, organizations and society, 28(2), pp. 127-168.

D’Onza G. (2008), Il sistema di controllo interno nella prospettiva del risk management, Milano, Giuffrè.

Greco G., Presti C., Marchi L. (2019). Il sistema di controllo interno: evoluzione e prospettive, in Greco G., Marchi L. (2019), Nuove prospettive su governance, audit, risk e performance, Torino, Giappichelli

Marchi L. (2011), L'evoluzione del controllo di gestione nella prospettiva informative e gestionale esterna, Management Control, 3, pp. 5-16. Doi: 10.3280/MACO2011-003001

Marchi L. (2019), La creazione e la misurazione del valore: dalla prospettiva finanziariaalla prospettiva economico-sociale, Lectio Magistralis

Messner M. (2016). Does industry matter? How industry context shapes management accounting practice. Management Accounting Research, 31, pp. 103-111.

Potito L. (2018), Prefazione: Il pensiero (contabile) declina, la prassi avanza, Milano, FrancoAngeli, pp.11-20.

Quagli A. (2001). L'informativa volontaria nel bilancio: ruolo, modalità e recenti tendenze, Revisione contabile, 41. 